(2) Open Access Full Text Article

\title{
Impact of baseline blood pressure on the magnitude of blood pressure lowering by nifedipine gastrointestinal therapeutic system: refreshing the Wilder's principle
}

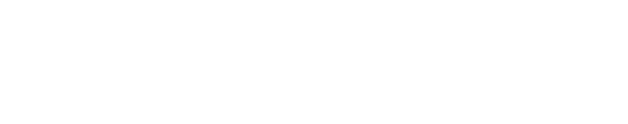

Haijuan Hu

Jidong Zhang

Yan Wang

Zejun Tian

Demin Liu

Guangming Zhang

Guoqiang Gu

Hongmei Zheng

Ruiqin Xie

Wei Cui

Department of Cardiology, The Second Hospital of Hebei Medical University, Shijiazhuang, Hebei, People's Republic of China
Correspondence: Wei Cui

Department of Cardiology, The Second Hospital of Hebei Medical University, No. 215 Hepingxi Road, Shijiazhuang, Hebei, People's Republic of China Tel/fax +8631166002115

Email cuiwei@medmail.com.cn
Background: The objective of the study was to investigate the relationship between baseline blood pressure (BP) and the magnitude of BP reduction in patients with essential hypertension treated with nifedipine gastrointestinal therapeutic system (NGTS).

Methods and patients: One hundred and thirty-eight patients with essential hypertension were enrolled in this prospective, single-arm, open-label study. NGTS was administered for 24 weeks to achieve target BP of 140/90 $\mathrm{mmHg}$. The dose could be uptitrated to $60 \mathrm{mg} / \mathrm{d}$ in case of unsatisfactory BP reduction after 4-week treatment. Home blood pressure measurement was recorded through the initial 1-14 days, and office BP and heart rate were evaluated at 2, 4, 8, 12, and 24 weeks. Results: One hundred and seventeen patients (84.8\%) completed the study, and their average BP decreased by $19.0 / 11.3 \mathrm{mmHg}$ after 24 weeks. The reduction of either systolic or diastolic BP was positively correlated with baseline BP at weeks 2,4 , or 24 after treatment $(r=0.603-0.762$, all $p<0.05)$. The maximal BP reduction was observed in $83 \%$ of patients at 4 weeks of treatment even though the dose of nifedipine remained unchanged ( $30 \mathrm{mg} / \mathrm{day})$.

Conclusion: These findings show that BP reduction is greatly influenced by the baseline level. Patients with high baseline BP had maximum reduction after treatment with NGTS, and the maximal antihypertensive efficacy of NGTS could appear even at 4 weeks after treatment initiation.

Keywords: baseline blood pressure, hypertension, nifedipine gastrointestinal therapeutic system

\section{Introduction}

Blood pressure (BP) management is becoming more challenging as it engages patients, families, care providers, and health care professionals. Early target BP approach is one of the important challenges in treatment of hypertension. Previous studies and guidelines recommend initial combination therapy for early BP control, particularly for patients with BP above 160/100 $\mathrm{mmHg}$ or BP $20 \mathrm{mmHg}$ above target BP. ${ }^{1-7}$ The rationale for this recommendation is that any standard dose of a single agent will lower BP by $10 / 5 \mathrm{mmHg}$, while any combination of standard dose of two antihypertensive drugs will reduce BP by $20 / 10 \mathrm{mmHg} .{ }^{8,9}$ It has been reported that BP reduction with antihypertensive drugs is directly related to the baseline $\mathrm{BP},{ }^{10,11}$ challenging the concept of initial combined administration. This effect is in concordance with the wellknown Wilder's principle that states the "direction of response of body function to any agent depends to a large degree on the initial value of that function". ${ }^{12}$ Few studies

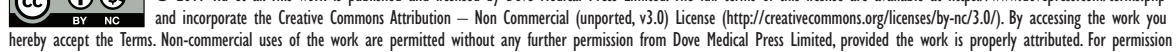
hereby accept the Terms. Non-commercial uses of the work are permitted without any further permission from Dove Medicat 
have reported correlation between initial $\mathrm{BP}$ and response to treatment. ${ }^{13,14}$ In addition, many previous studies have also shown that the maximum antihypertensive effect of antihypertensive drugs usually appears at 4-8 weeks after treatment with no relation with the half-life period of those antihypertensive drugs. ${ }^{15}$ The EARLY trial was a prospective, single-group, single-center, and open-label study designed to evaluate the impact of nifedipine gastrointestinal therapeutic system (NGTS) on pulse wave velocity (PWV) in Chinese hypertensive patients (Chinese Clinical Trial Registry: ChiCTR-ONC-13004063). The study also provided daily home blood pressure measurement (HBPM) data within 2 weeks of treatment, and single-dose NGTS was used within 4 weeks according to the study protocol. ${ }^{16}$ Therefore, we further analyzed the EARLY data to provide evidence about influence of baseline $\mathrm{BP}$ on the $\mathrm{BP}$ reduction and the time to achieve the maximum BP-lowing effect.

\section{Methods and patients Study design and treatment regimens}

This single-center, single-group, prospective, phase IV clinical trial enrolled 138 newly diagnosed hypertension patients. Hypertension diagnosis had to be confirmed by 24-hour ambulatory BP monitoring with SunTech's Oscar2 ${ }^{\mathrm{TM}}$ monitoring system (Sun Tech, Morrisville, NC, USA). NGTS was administered at a dose of $30 \mathrm{mg} / \mathrm{d}$, with an office BP target of 140/90 mmHg. Dose uptitration of NGTS up to $60 \mathrm{mg} / \mathrm{d}$ was allowed in case of unsatisfactory BP reduction after 4 weeks. Patients were visited at 2, 4, 8, 12, and 24 weeks to record patients' systolic blood pressure (SBP) and diastolic blood pressure (DBP), heart rate, and necessary lab studies.

\section{Study protocol HBPM protocol}

Home BP was measured with an Omron electronic BP (Omron Healthcare, Kyoto, Japan) measuring device in the morning and in the evening every day within the first 14 days of the trial. Three consecutive recordings were obtained with a gap of 1 minute and the last two recordings were averaged.

\section{OBPM protocol}

Office BP was measured by the researcher who was in charge of the follow-up visits in the office, with the same brand's electronic BP measuring device.

\section{Patient population}

This study enrolled 138 patients with essential hypertension treated in the Second Hospital of Hebei Medical University from December 2013 to December 2014. Inclusion criteria were as follows: patients aged 18-75 years who signed informed consent; diagnosed as hypertensive by 24-hour ambulatory BP monitoring with clinic SBP $\geq 140 \mathrm{mmHg}$, or DBP $\geq 90 \mathrm{mmHg}$; and body mass index $<33 \mathrm{~kg} / \mathrm{m}^{2}$. The exclusion criteria were pregnant women, persons who participated in drug clinical trials within 3 months, patients known to be intolerant of NGTS or its components, taking any other antihypertensive drugs; patients with secondary hypertension; patients with serious cardiovascular diseases including history of cardiogenic shock, myocardial infarction or unstable angina within 6 months, severe valvular heart disease, past or current severe cardiac arrhythmias, stroke within the past 12 months; and patients with type 1 or type 2 diabetes. Patients with AST/ALT above three times of the upper limit of normal, or with creatinine level above $2.0 \mathrm{mg} / \mathrm{dL}$, malignant disease, alcohol abuse, or drug abuse were also excluded. This study was approved by the Ethics Committee of the Second Hospital of Hebei Medical University, People's Republic of China. All the patients signed the written informed consent form before enrollment and were screened for inclusion in the study. Current smoker was defined as a person who smoked more than 100 cigarettes in their lifetime and has smoked in past 30 days. A drinker is defined as a person who drinks alcoholic beverages.

\section{Statistical analysis}

Statistical analysis was performed using SPSS statistical software (version 17.0, SPSS Inc, Chicago, IL, USA). Continuous variables were expressed as mean \pm standard deviation, and categorical data were expressed as percentage under normal data distribution. Repeated measures one-way analysis of variance was applied for comparing parameters at baseline and 4, 12, and 24 weeks. Pearson linear regression analysis was performed to determine the correlation between the baseline $\mathrm{BP}$ and $\mathrm{BP}$ reduction at the aforementioned time points. A $p$-value of $<0.05$ was considered to be statistically significant.

\section{Results \\ Baseline characteristics}

Among the 138 subjects, 21 (15.2\%) withdrew from the study (16 were lost to follow-up, three due to intolerable headache, and two due to unsatisfied antihypertensive effect). Finally 117 subjects completed this study, including 71 males (60.7\%) and 46 females (39.3\%); age $48.4 \pm 11.9$ years. Of the 117 patients, 97 patients received $30 \mathrm{mg}$ NGTS once daily (OD) through the study period. Baseline characteristics of the study population are shown in Table 1. 
Table I Demographics of the study cohort

\begin{tabular}{ll}
\hline Variable & Total $(\mathbf{n}=\mathbf{I}$ I 7) \\
\hline Male & $71(60.68 \%)$ \\
Age (years) & $48.4 \pm I I .9$ \\
BMI $\left(\mathrm{kg} / \mathrm{m}^{2}\right)$ & $26.0 \pm 2.9$ \\
Current smoker & $25(21.37 \%)$ \\
Drinker & $7(5.98 \%)$ \\
GLU $(\mathrm{mmol} / \mathrm{L})$ & $5.32 \pm 0.78$ \\
TG $(\mathrm{mmol} / \mathrm{L})$ & $1.88 \pm 1.00$ \\
$\mathrm{CHOL}(\mathrm{mmol} / \mathrm{L})$ & $4.70 \pm 0.89$ \\
$\mathrm{HDL}(\mathrm{mmol} / \mathrm{L})$ & $1.42 \pm 0.4 \mathrm{I}$ \\
$\mathrm{LDL}(\mathrm{mmol} / \mathrm{L})$ & $2.54 \pm 0.85$
\end{tabular}

Notes: Data are presented as mean \pm standard deviation unless otherwise specified.

Abbreviations: BMI, body mass index; GLU, glucose; TG, triglyceride; $\mathrm{CHOL}$, cholesterol; HDL, high-density lipoprotein; LDL, low-density lipoprotein.

\section{Impact of NGTS on SBP and DBP}

SBP, DBP, and pulse pressure (PP) were significantly lower at $2,4,8,12$, and 24 weeks after treatment with NGTS compared with baseline $(p<0.01)$, while nonsignificant changes were observed in SBP, DBP, and PP between values after 4, 12, and 24 weeks of treatment $(p>0.05)$, indicating that NGTS has reached its maximal antihypertensive effect in 4 weeks after treatment (Figure 1). There was no significant change in heart rate before and after antihypertensive therapy $(p>0.05)$.

In order to clarify the possible causes for BP reaching the maximal reduction in the 4 th week, the study population was divided into $30 \mathrm{mg}(\mathrm{n}=97)$ and $60 \mathrm{mg}(\mathrm{n}=20)$ groups according to the doses of NGTS. The results showed clearly that the maximal BP reduction was achieved from the 4 th week after treatment in the $30 \mathrm{mg}$ group, although

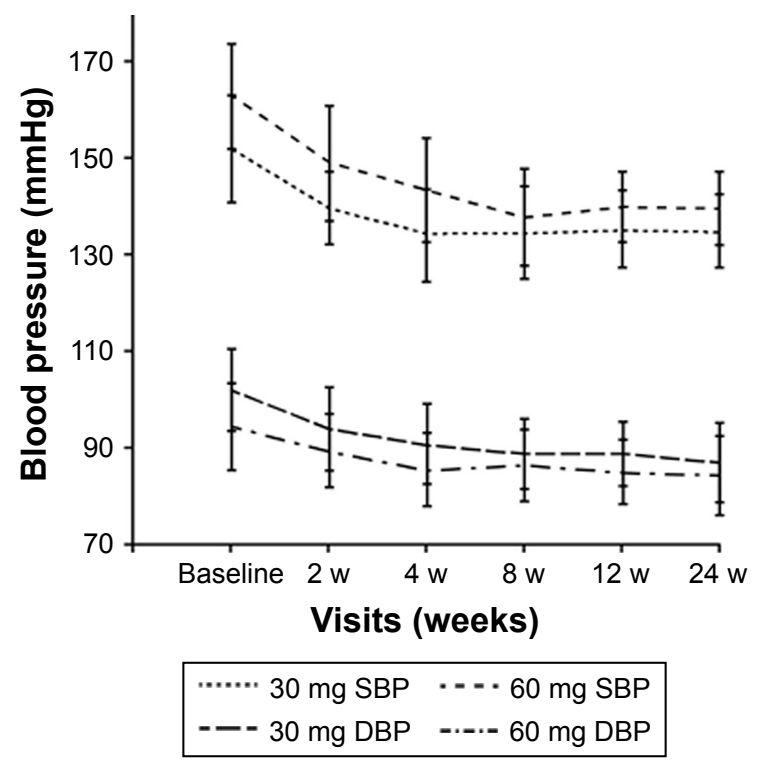

Figure I SBP and DBP changes in 30 and $60 \mathrm{mg}$ nifedipine group by visit. Abbreviations: SBP, systolic blood pressure; DBP, diastolic blood pressure. the NGTS dose remained unchanged throughout the study period (Figure 1).

\section{Impact of baseline SBP on SBP reduction}

To observe the SBP reduction at various baseline levels with NGTS, the study population was grouped according to baseline SBP (Figure 2). The results showed that the mean SBP reduction at weeks 2,4 , and 24 after treatment was positively correlated with baseline BP $(r=0.689,0.603$, and 0.762 , respectively, $p<005$ Figure 3 ). Furthermore, the baseline SBP affected the timing of maximal SBP reduction, even for the patients who continued to receive $30 \mathrm{mg}$ NGTS (Tables 2 and 3). These results suggest that the mean SBP reduction by NGTS is significantly affected by baseline BP, and can be accurately predicted by baseline $\mathrm{BP}$ level. The higher the baseline $\mathrm{BP}$, the later the maximal antihypertensive effect appears.

\section{Impact of baseline DBP on mean DBP reduction}

To observe the mean DBP reduction at various baseline levels with NGTS, we divided the enrolled patients into different groups according to baseline DBP (Figure 4). The results showed that DBP reduction at weeks 2, 4, and 24 after treatment was also positively correlated with baseline DBP $(r=0.599,0.627$, and 0.543 , respectively, $p<0.05$; Figure 5).

\section{Changes in HBPM after NGTS treatment within initial 14 days}

In order to further observe the timing of maximal BP reduction after treatment with NGTS, the daily changes in

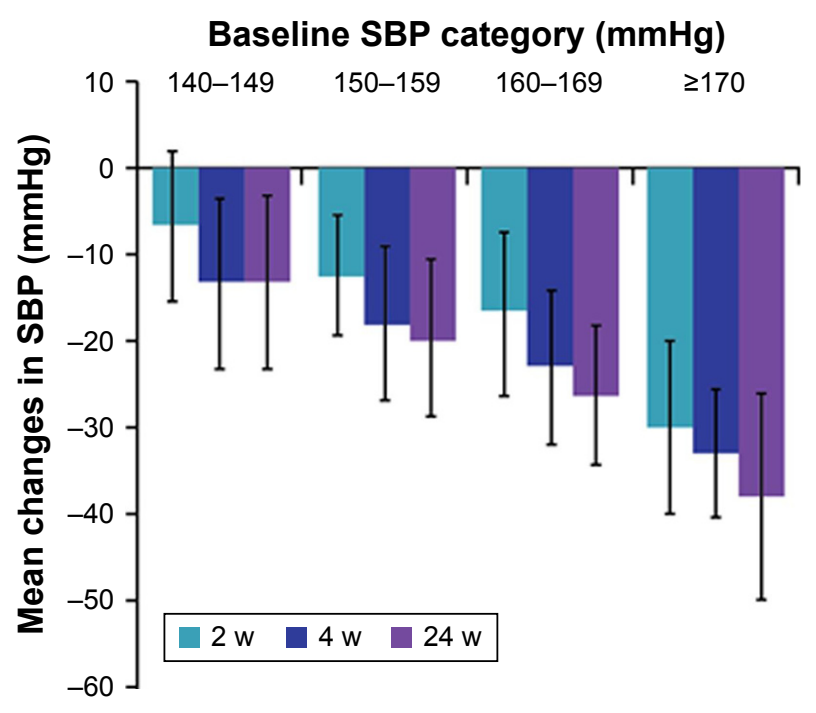

Figure 2 Mean reduction in SBP achieved with nifedipine from initial to final visit $(n=117)$, according to baseline SBP category. Abbreviation: SBP, systolic blood pressure. 

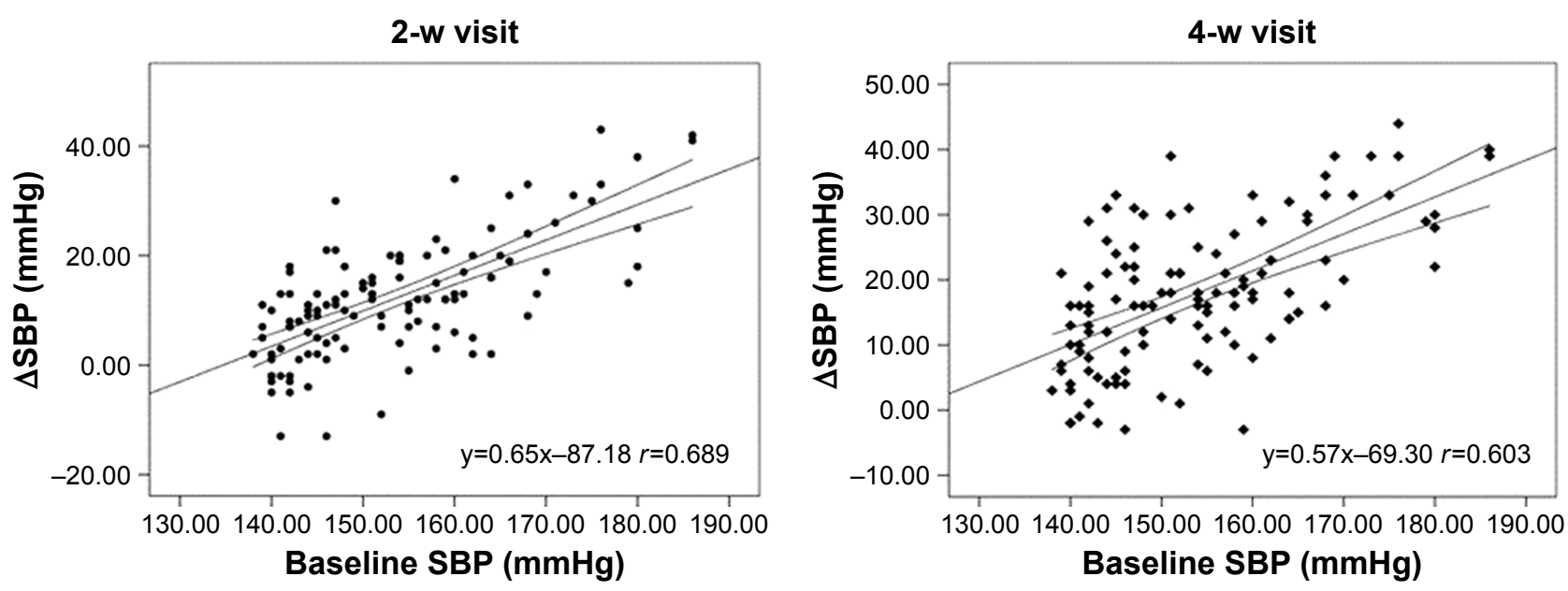

24-w visit

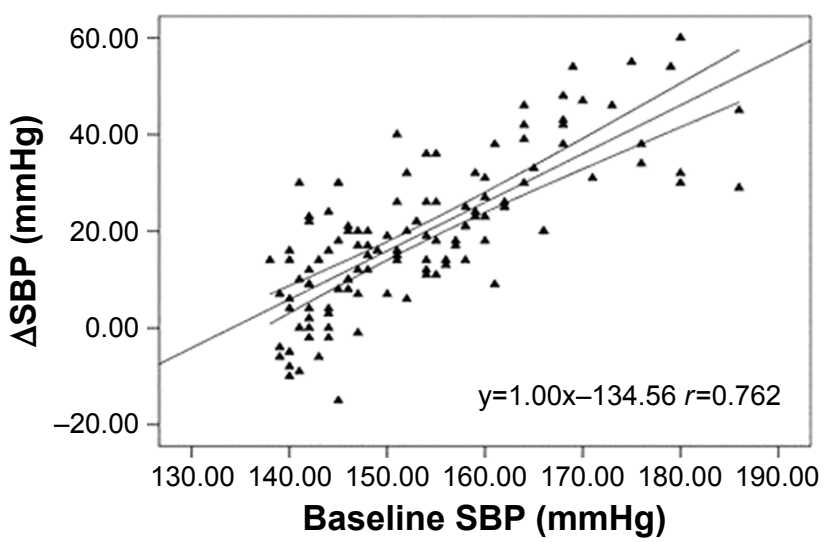

Figure 3 The scatter diagram of mean reduction and baseline SBP after the 2, 4, and 24 weeks of treatment. Abbreviation: SBP, systolic blood pressure.

HBPM within the initial 14 days were documented. The results showed that maximal SBP/DBP reduction occurred on the 3rd day of treatment and remained steady throughout 14 days (Figure 6). The mean SBP at baseline and 14th day was $144.27 \pm 12.06 \mathrm{mmHg}$ and $134.90 \pm 10.66 \mathrm{mmHg}$, respectively. The mean DBP at baseline and 14th day was $91.00 \pm 9.73 \mathrm{mmHg}$ and $85.58 \pm 9.03 \mathrm{mmHg}$, respectively.

\section{Discussion}

The $10 / 5$ or $20 / 10$ concept of antihypertensive treatment is that any standard dose of a single antihypertensive drug will lower BP by $10 / 5 \mathrm{mmHg}$, while any combination of standard dose of two antihypertensive drugs will reduce BP by $20 / 10 \mathrm{mmHg} .{ }^{8,9,17}$ However, many previous studies have reported that BP-lowering effect of antihypertensive drugs was related to baseline level of BP, and hence the concept of starting combined administration is challenged. AdADOSE study shows that, whether patients are under initial treatment or already taking some antihypertensive drugs, BP reduction is obviously related to baseline BP. The higher the baseline $\mathrm{BP}$, the greater the reduction in BP. ${ }^{18}$ Ueng et al studied 14,266 patients with hypertension treatment with NGTS

Table 2 SBP and DBP achieved with nifedipine 30 or $60 \mathrm{mg}$ once daily from initial visit to follow-up ( $\mathrm{n}=1 \mathrm{I} 7)$

\begin{tabular}{lllllll}
\hline & Baseline & 2 weeks & 4 weeks & 8 weeks & I2 weeks & 24 weeks \\
\hline SBP $(\mathrm{mmHg})$ & $153.0 \pm I I .8$ & $141.4 \pm 8.9$ & $135.9 \pm 10.1$ & $134.4 \pm 8.0$ & $134.1 \pm 8.5$ & $134.0 \pm 9.9$ \\
DBP $(\mathrm{mmHg})$ & $94.7 \pm 9.5$ & $87.0 \pm 8.4$ & $84.9 \pm 7.6$ & $84.1 \pm 7.3$ & $83.9 \pm 7.9$ & $83.4 \pm 9.3$ \\
PP & $58.9 \pm 10.8$ & $54.3 \pm 7.5$ & $50.9 \pm 7.6$ & $50.3 \pm 7.0$ & $50.2 \pm 7.1$ & $50.6 \pm 7.7$ \\
HR & $74.1 \pm 10.4$ & $76.0 \pm 8.9$ & $75.4 \pm 10.6$ & $75.6 \pm 11.2$ & $75.8 \pm 10.8$ & $76.1 \pm 11.5$ \\
\hline
\end{tabular}

Note: Data are presented as mean \pm standard deviation.

Abbreviations: SBP, systolic blood pressure; DBP, diastolic blood pressure; PP, pulse pressure; HR, heart rate. 
Table 3 Mean SBP and DBP achieved with nifedipine $30 \mathrm{mg}(\mathrm{n}=97)$ and $60 \mathrm{mg}(\mathrm{n}=20)$ once daily from initial visit to follow-up

\begin{tabular}{|c|c|c|c|c|c|c|}
\hline & Baseline & 2 weeks & 4 weeks & 8 weeks & 12 weeks & 24 weeks \\
\hline \multicolumn{7}{|c|}{ SBP (mmHg) } \\
\hline $30 \mathrm{mg}$ & $151.7 \pm 1 \mid .0$ & $139.8 \pm 7.4$ & $134.3 \pm 9.3$ & $134.6 \pm 9.6$ & $135.4 \pm 8 . \mid$ & $135.1 \pm 7.9$ \\
\hline $60 \mathrm{mg}$ & $163.1 \pm 10.8$ & $149.1 \pm 1 \mid .7$ & $143.5 \pm 10.6$ & $137.8 \pm 9.9$ & $139.9 \pm 7.1$ & | $39.8 \pm 7.6$ \\
\hline \multicolumn{7}{|c|}{$\mathrm{DBP}(\mathrm{mmHg})$} \\
\hline $30 \mathrm{mg}$ & $94.5 \pm 8.9$ & $89.5 \pm 7.6$ & $85.4 \pm 7.6$ & $86.7 \pm 7.4$ & $85 . I \pm 6.8$ & $84.5 \pm 8.1$ \\
\hline $60 \mathrm{mg}$ & $101.9 \pm 8.5$ & $94.3 \pm 8.6$ & $90.9 \pm 8.3$ & $88.8 \pm 7.2$ & $88.9 \pm 6.7$ & $87.3 \pm 8.3$ \\
\hline
\end{tabular}

Note: Data are presented as mean \pm standard deviation.

Abbreviations: SBP, systolic blood pressure; DBP, diastolic blood pressure.

and demonstrated that baseline BP has obvious effect on BP reduction. ${ }^{11}$ Similar results were reported in 1,466 hypertensive patients from Middle East. ${ }^{19}$ DISTINCT, an 8 week, double-blind, randomized study, showed that baseline BP significantly affected BP reduction amplitude for both monotherapy (NGTS or candesartan) or combined therapy (NGTS/ candesartan). ${ }^{20}$ However, these studies used more than one drug and multiple doses, so clear impact of baseline BP on the magnitude of BP reduction might not be attributed by drugs used or doses taken. Therefore, the magnitude of BP reduction might not be accurately predicted. In this study, we clearly demonstrated the impact of baseline $\mathrm{BP}$ on $\mathrm{BP}$ reduction magnitude by using NGTS monotherapy, which showed that $\mathrm{BP}$ reduction was positively correlated with baseline BP at the observed time points after treatment. The further analysis in patients who received single dose of NGTS (30 mg/day) throughout the study period also showed the same results. Our results are not in agreement with the antihypertensive 10/5 concept, and don't support the initial combination strategy for some selected hypertensive patients, which we think further study in larger clinical trials is highly recommended.

An interesting phenomenon in antihypertensive therapy is that the maximal antihypertensive effect of antihypertensive

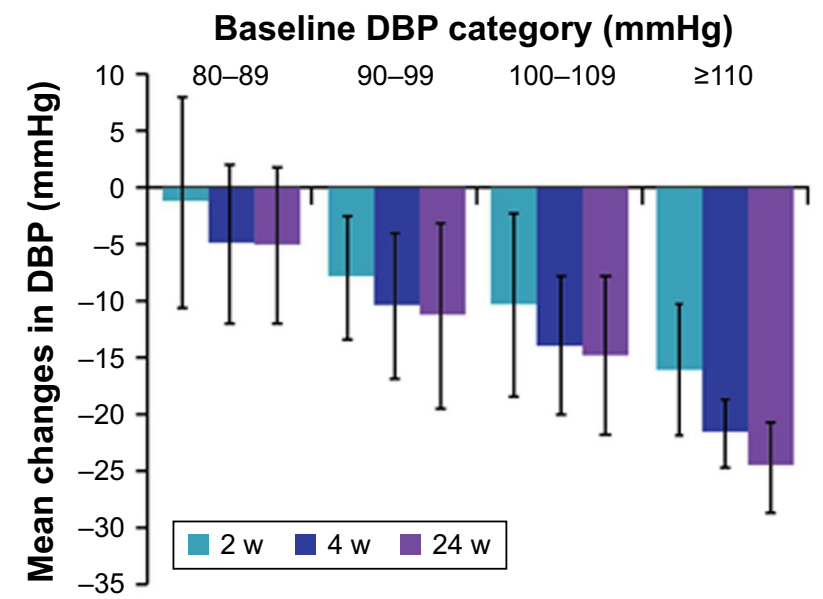

Figure 4 Mean reduction in DBP achieved with nifedipine from initial to final visit $(n=117)$ according to baseline DBP category.

Abbreviation: DBP, diastolic blood pressure. drugs appears around 4 weeks after treatment. ${ }^{15}$ The DISTINCT trial demonstrated that the maximal BP reduction amplitude was reached in the 4th week of treatment, either in NGTS or candesartan monotherapy, or in combination therapy groups. ${ }^{20}$ Similar results were seen with NGTS monotherapy or combination of amlodipine/valsartan. ${ }^{21}$ The TALENT trial even showed that the maximal BP reduction occurred 16 weeks after treatment in NGTS and telmisartan combination group. ${ }^{22}$ A post-marketing surveillance on NGTS in People's Republic of China showed that antihypertensive efficacy further declined as medication time is extended, in which $98.6 \%$ of the patients were using NGTS $30 \mathrm{mg} /$ day. ${ }^{10}$ Similar results was also reported in another study. ${ }^{11}$ Our current study also showed that the maximal antihypertensive efficacy of NGTS appeared 4 weeks after treatment initiation even at the same daily dose, and was positively correlated with baseline BP level. Daily HBPM results within 2 weeks of treatment showed stability in BP within first 3 days after NGTS treatment, which is consistent with previous study that nifedipine plasma concentration reached steady and consistent state after 3 doses (OD) of NGTS. ${ }^{23}$ Other studies reported correlation between first dose of nifedipine and responsiveness after first few weeks of treatment and height of pre-treatment BP. ${ }^{24}$ In view of the timing of maximal BP reduction is obviously in disagreement with the timing of steady blood concentration, we believe that there is a delayed effect in antihypertensive treatment. It can be seen from this study that the delayed antihypertensive effect was about $8 \mathrm{mmHg}$.

Delayed effects of antihypertensive therapy may have various mechanisms. One of these possible explanations might be that the antihypertensive therapy could improve arterial structure and function. Schiffrin showed that treatment with NGTS for 1 year with good BP control could significantly improve the structure and function of arteriole, while atenolol did not exhibit similar vascular protection although BP could also be controlled at similar level. ${ }^{25}$ Another study reported that nifedipine could reduce carotid and femoral intima-media 

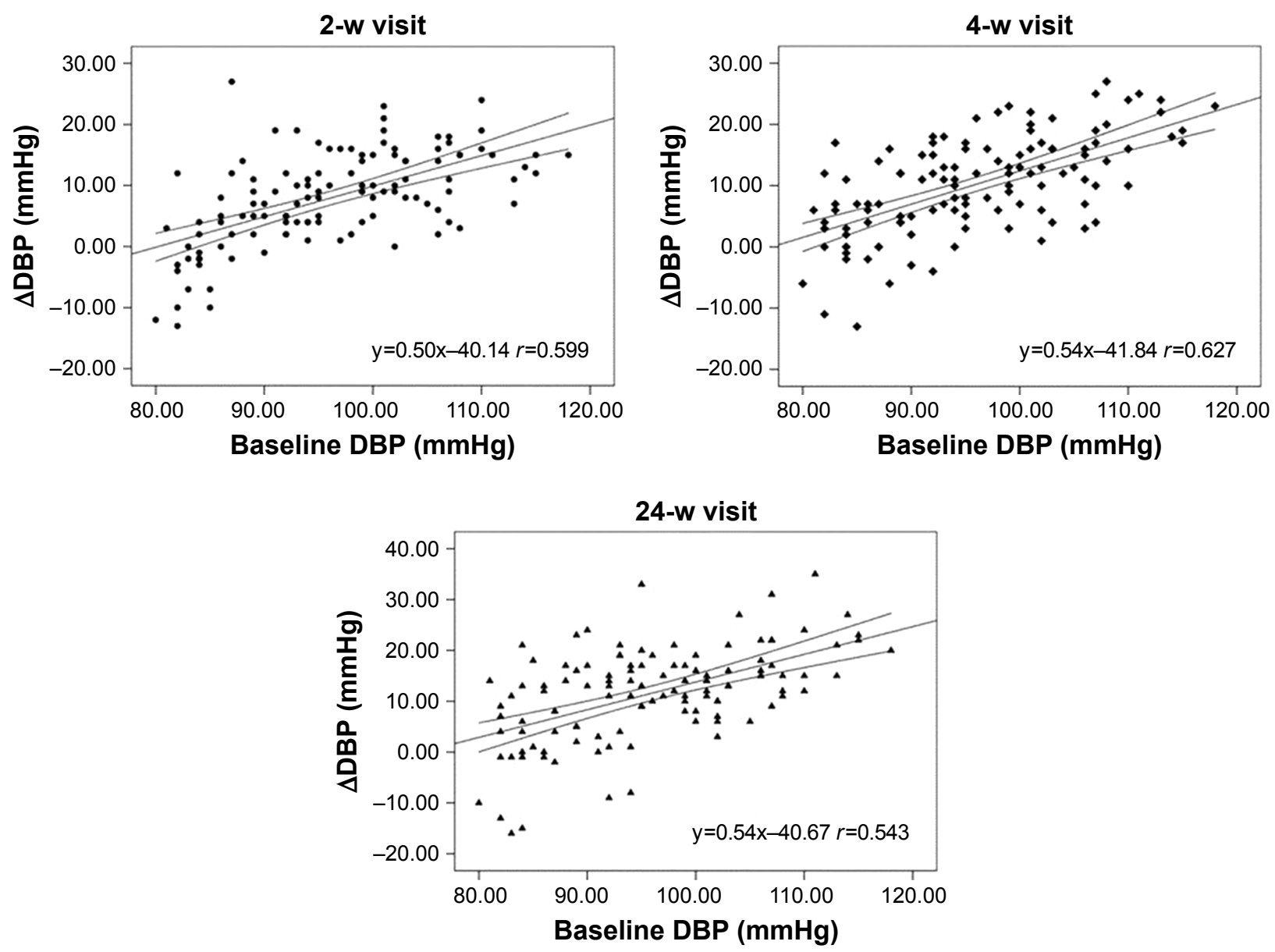

Figure 5 The scatter diagram of mean reduction and baseline DBP after 2, 4, and 24 weeks of treatment. Abbreviation: DBP, diastolic blood pressure.

thickness in newly diagnosed hypertensive patients. ${ }^{26}$ Significant improvement in vascular endothelial function was reported by Sudano et al in patients with hypertension by the use of NGTS for 24 weeks. ${ }^{27}$ Our previous study also

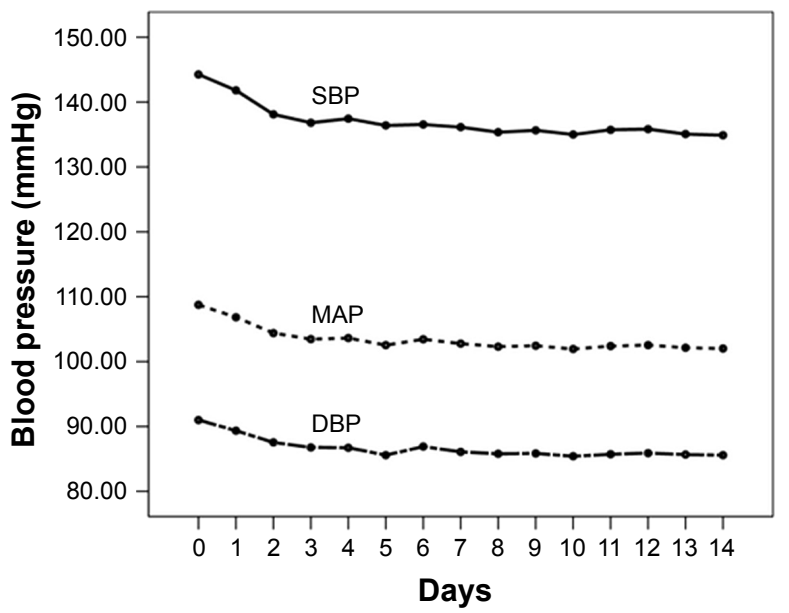

Figure 6 Changes in HBPM after 14 days of NGTS treatment.

Abbreviations: HBPM, home blood pressure measurement; NGTS, nifedipine gastrointestinal therapeutic system; SBP, systolic blood pressure; MAP, mean arterial pressure; DBP, diastolic blood pressure. showed that treatment with NGTS for 24 weeks can persistently improve brachial-ankle PWV, which was independent of BP reduction. ${ }^{16}$ In their paper, Sudano et al also showed an interesting phenomenon in which NGTS significantly reduced patients' BP (from 164.4 $\pm 5.4 / 103.8 \pm 4.4 \mathrm{mmHg}$ before treatment to $126.9 \pm 9.5 / 81.2 \pm 2.4 \mathrm{mmHg}$ after treatment) for about 1 year. After NGTS was withdrawn for 3 days, the BP returned to $154.0 \pm 6.3 / 96.9 \pm 3.3 \mathrm{mmHg}$ instead of the baseline level. ${ }^{27}$ The study results by Sudano et al further suggest that longterm hypertensive treatment improve vascular structure and function, so the BP is still lower than the level before treatment even if after drug withdrawal. Therefore, the most likely mechanism for the delayed effect of antihypertensive therapy is the improvement of arterial structure and function by antihypertensive therapy. It is still not clear whether different antihypertensive drugs have different delayed antihypertensive effects.

One of the recommended strategies nowadays in antihypertensive therapy is to shorten the time of drug titration in order to get BP goal early. The Eighth Joint National Committee suggested that titration period may be 1 month. ${ }^{3} \mathrm{ACC} / \mathrm{AHA}$ and ASH Hypertension Guidelines recommend that drug 
adjustment can be as early as 2 weeks if BP is still higher than targeted BP after treatment. ${ }^{2,7}$ In this study, our daily HBPM of initial 14 days showed that BP can generally reach steady level within first 3 days after taking NGTS, which suggests titration period for NGTS might be as short as 3 days because the $\mathrm{BP}$ reduction could be predicted at 3 days after treatment plus the delayed effect (about $8 \mathrm{mmHg}$ ). This may provide a simple and faster drug titration approach for clinicians.

There are a few limitations of the study. Firstly, as evident from the literature there is a statistical correlation between baseline BP and magnitude of BP response to any treatment including placebo. ${ }^{13,28}$ However, in our study we do not have placebo effect, which might be a confounding factor in accurately assessing the magnitude of the antihypertensive response to drug treatment. Secondly, this may also be a confounding factor for calculating the time for achieving the maximal effect of antihypertensive drug treatment.

\section{Conclusion}

This single-center, single-group, prospective, phase IV clinical trial revealed that there might be a delayed antihypertensive effect of NGTS. Further, this current study provides evidence that $\mathrm{BP}$ reduction is dependent on baseline level and that the maximal antihypertensive efficacy of NGTS could appear even at 4 weeks after treatment initiation.

\section{Acknowledgments}

The authors would like to acknowledge Dr Anuradha Nalli and Dr Amit Bhat (Indegene Private. Ltd., Bangalore) for providing medical writing and editorial support in the preparation of the manuscript.

\section{Disclosure}

The authors report no conflicts of interest in this work.

\section{References}

1. Cheng HM, Chiang CE, Chen CH. The Novelty of the 2015 Guidelines of the Taiwan Society of Cardiology and the Taiwan Hypertension Society for the Management of Hypertension. Pulse (Basel). 2015;3(1):29-34.

2. Go AS, Bauman MA, Coleman King SM, et al. An effective approach to high blood pressure control: a science advisory from the American Heart Association, the American College of Cardiology, and the Centers for Disease Control and Prevention. J Am Coll Cardiol. 2014;63(12): 1230-1238.

3. James PA, Oparil S, Carter BL, et al. 2014 evidence-based guideline for the management of high blood pressure in adults: report from the panel members appointed to the Eighth Joint National Committee (JNC 8). JAMA. 2014;311(5):507-520.

4. Leung AA, Nerenberg K, Daskalopoulou SS, et al; CHEP Guidelines Task Force. Hypertension Canada's 2016 Canadian Hypertension Education Program Guidelines for Blood Pressure Measurement, Diagnosis, Assessment of Risk, Prevention, and Treatment of Hypertension. Can J Cardiol. 2016;32(5):569-588.
5. Mancia G, Fagard R, Narkiewicz K, et al. 2013 ESH/ESC guidelines for the management of arterial hypertension: the Task Force for the Management of Arterial Hypertension of the European Society of Hypertension (ESH) and of the European Society of Cardiology (ESC). Eur Heart J. 2013;34(28):2159-2219.

6. Wald DS, Law M, Morris JK, Bestwick JP, Wald NJ. Combination therapy versus monotherapy in reducing blood pressure: meta-analysis on 11,000 participants from 42 trials. Am J Med. 2009;122(3):290-300.

7. Weber MA, Schiffrin EL, White WB, et al. Clinical practice guidelines for the management of hypertension in the community a statement by the American Society of Hypertension and the International Society of Hypertension. J Hypertens. 2014;32(1):3-15.

8. Chiang CE, Wang TD, Ueng KC, et al. 2015 guidelines of the Taiwan Society of Cardiology and the Taiwan Hypertension Society for the management of hypertension. J Chin Med Assoc. 2015;78(1):1-47.

9. Law MR, Wald NJ, Morris JK, Jordan RE. Value of low dose combination treatment with blood pressure lowering drugs: analysis of 354 randomised trials. BMJ. 2003;326(7404):1427.

10. Runlin G, Junren Z, Guozhang L, et al. Efficacy and safety of nifedipine GITS in Asians with hypertension: results of a post-marketing surveillance study in China. Clin Drug Investig. 2007;27(8):565-572.

11. Ueng KC, Ningling S, El Maksod A, Hung KY, Yuehui Y. Efficacy and tolerability of long-acting nifedipine GITS/OROS monotherapy or combination therapy in hypertensive patients: results of a 12-week international, prospective, multicentre, observational study. Clin Drug Investig. 2011;31(9):631-642.

12. Messerli FH, Bangalore S, Schmieder RE. Wilder's principle: pretreatment value determines post-treatment response. Eur Heart $J$. 2015;36(9):576-579.

13. Gill JS, Zezulka AV, Beevers DG, Davies P. Relation between initial blood pressure and its fall with treatment. Lancet. 1985;1(8428):567-569.

14. Sumner DJ, Howie K, Aitchison T, Elliott HL, Reid JL. Blood pressure and correlations. Lancet. 1985;325(8437):1110-1111.

15. Lasserson DS, Buclin T, Glasziou P. How quickly should we titrate antihypertensive medication? Systematic review modelling blood pressure response from trial data. Heart. 2011;97(21):1771-1775.

16. Zhang J, Wang Y, Hu H, et al. Early intervention of long-acting nifedipine GITS reduces brachial-ankle pulse wave velocity and improves arterial stiffness in Chinese patients with mild hypertension: a 24-week, single-arm, open-label, prospective study. Drug Des Devel Ther. 2016; 10:3399-3406.

17. Zusman R. Patients with uncontrolled hypertension or concomitant hypertension and benign prostatic hyperplasia. Clin Cardiol. 2004;27(2):63-69.

18. Motaweih AK, Usova E, Hussain W, Dello Z, Schmidt B, Petri T. Effectiveness of combination therapy with nifedipine GITS: a prospective, 12-week observational study (AdADOSE). BMC Cardiovasc Disord. 2015;15:35.

19. Ghoneim RA, Omar AK, Sebastian V, et al. Long-acting nifedipine for hypertensive patients in the Middle East and Morocco: observations on efficacy and tolerability of monotherapy or combination therapy. Integr Blood Press Control. 2013;6:49-57.

20. Kjeldsen SE, Cha G, Villa G, Mancia G; DISTINCT Investigators. Nifedipine GITS/candesartan combination therapy lowers blood pressure across different baseline systolic and diastolic blood pressure categories: DISTINCT study subanalyses. J Clin Pharmacol. 2016;56(9): $1120-1129$.

21. Wang JG, Zeng WF, He YS, et al; EXAM Investigators. Valsartan/ amlodipine compared to nifedipine GITS in patients with hypertension inadequately controlled by monotherapy. Adv Ther. 2013;30(8): 771-783.

22. Mancia G, Parati G, Bilo G, Choi J, Kilama MO, Ruilope LM; TALENT investigators. Blood pressure control by the nifedipine GITS-telmisartan combination in patients at high cardiovascular risk: the TALENT study. J Hypertens. 2011;29(3):600-609.

23. Chung M, Reitberg DP, Gaffney M, Singleton W. Clinical pharmacokinetics of nifedipine gastrointestinal therapeutic system. A controlledrelease formulation of nifedipine. Am J Med. 1987;83(6B):10-14. 
24. Donnelly R, Elliott HL, Meredith PA, Kelman AW, Reid JL. Nifedipine: individual responses and concentration-effect relationships. Hypertension. 1988;12(4):443-449.

25. Schiffrin EL. Structure and function of small arteries of essential hypertensive patients following chronic treatment with once-a-day nifedipine. Cardiology. 1997;88(Suppl 3):20-26.

26. Terpstra WF, May JF, Smit AJ, de Graeff PA, Crijns HJ. Effects of nifedipine on carotid and femoral arterial wall thickness in previously untreated hypertensive patients. Blood Press Suppl. 2003;1:22-29.
27. Sudano I, Virdis A, Taddei S, et al. Chronic treatment with long-acting nifedipine reduces vasoconstriction to endothelin-1 in essential hypertension. Hypertension. 2007;49(2):285-290.

28. MRC trial of treatment of mild hypertension: principal results. Medical Research Council Working Party. Br Med J (Clin Res Ed). 1985;291(6488):97-104.

\section{Publish your work in this journal}

Drug Design, Development and Therapy is an international, peerreviewed open-access journal that spans the spectrum of drug design and development through to clinical applications. Clinical outcomes, patient safety, and programs for the development and effective, safe, and sustained use of medicines are the features of the journal, which has also been accepted for indexing on PubMed Central. The manuscript management system is completely online and includes a very quick and fair peer-review system, which is all easy to use. Visit http://www.dovepress.com/testimonials.php to read real quotes from published authors.

Submit your manuscript here: http://www.dovepress.com/drug-design-development-and-therapy-journal 